\title{
Study on Security Risk Propagation of Immune Mechanism under Complex Network Background
}

\author{
Lu Cailin \\ School of Information Technology and Engineering, Jinzhong University, Jinzhong, \\ Shanxi, China \\ 13453296628@163.com
}

\begin{abstract}
The economic situation highlights the significant role of the application of computer in human society since the 21st century, especially since 2015. This thesis probes into the security risk propagation of immune mechanism under complex network background. Focusing on the issue of network security under network background, the thesis deploys the analysis by adopting the mechanism model of immune mechanism, immunizing parts of the nodes, establishing stochastic immune mechanism of security risk and introducing stochastic immune mechanism propagation model of security risk. To further study the security risk propagation of immune mechanism under complex network background, the thesis presents the model rules, introduces the security risk acquaintance immune mechanism and establishes models of acquaintances immune mechanism. Finally, attributing to the simulation experiments, the simulation results, the characteristics of security risk propagation model, and the study of stochastic immune mechanism propagation model and acquaintance immune mechanism stochastic model, the thesis reaches the conclusion that at the initial stage of propagation, the die-out rate of the security risk is inversely proportional to the propagation rate.
\end{abstract}

Keywords: complex networks, security risks, stochastic immune mechanism propagation model, acquaintance immune mechanism propagation model

\section{Introduction}

As for the complex network, initially scientists study always uses the network which is a formation of the point and the edge to abstract the complexity of real situation. But this method will have certain limitations that there is no way to explain the risk communication of immune mechanism. Until 1999, Barbra and Albert found a feature called scale-free networks. Which improved the characteristics of the complex network's structure, including the delicate relationship between the various mechanical characteristics and the characteristics of the network structure? This paper can more practical use network theory to explain the risk propagation of immune mechanism.

To meet the needs of development of the society, the perfection of current internet situation and the satisfaction of people's hope for technological security, studies on security risk propagation of immune mechanism are becoming increasingly important [1]. In the aspect of immune mechanism, some scholars and professors, including Song Yurong, have proposed the stochastic immune mechanism and established models with immune mechanism after some studies under the presumption that some complex network malicious software may be spread by the cellular automata in real life. However, this model neglected the condition of health status. Based on the researches conducted by the former scholars, this study established security risk immune mechanism. And by referring to the above-mentioned two kinds of models, the thesis further studies and completes the issue of security risk propagation of immune mechanism under the complex network [2]. 


\section{Models of Security Risk Propagation based on Immune Mechanism}

\subsection{Complex Networks}

A variety of network forms with high complexity exist in our life, including the interpersonal relationship network, the neural network within the body, telephone network, wireless network and the risk transmission network, all known as "complex network" which has become one of the important parts in the study of the important subject. Complex networks can have a variety of forms, such as a directed graph, weighted graph, etc. However, the structure has the similarity basically no matter how the method to indicate that. Complex networks can be divided into three types: small world networks, random networks and scale free networks. Watts defines the following topological features as small world characteristics:

$$
\left\{\begin{array}{l}
C>>C_{\text {random }} \\
L \geq \text { Lrandom }
\end{array}\right.
$$

Where: $\mathrm{C}$ is clustering coefficient; $\mathrm{L}$ is the average distance network; subscripts means random network.

Combining complex network cluster structure analysis to describe the intrinsic link between the various nodes of the immune mechanism security risk propagation. In this paper, we use directed graph to represent the mechanism of security risk propagation. There are many clustering methods for complex networks. In this paper, a variety of methods are listed in the form of graphs, and the classification is carried out, as shown in Figure 1:

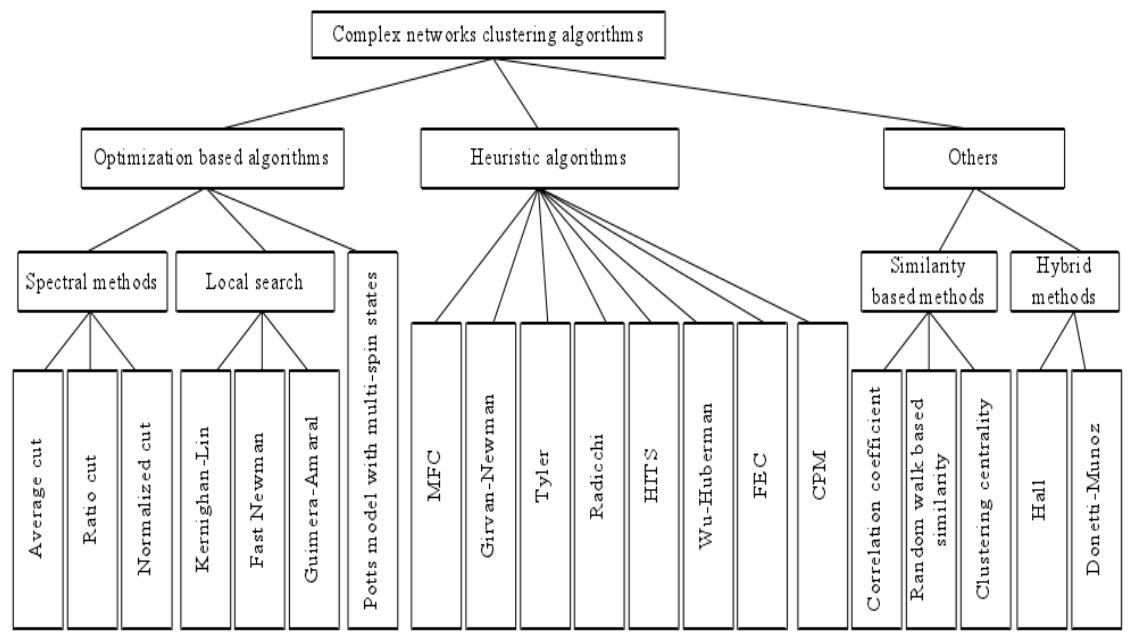

Figure 1. Classification Chart of Complex Network Clustering Algorithms

\subsection{Propagation Model of Stochastic Immune Mechanism}

(1) Mechanism analysis of the model

The so-called stochastic immune mechanism refers to immunizing parts of the selected nodes, and then introducing the immunized nodes into security risk propagation model studied in this thesis under complex network circumstance. Among which, the node statuses are presented as follow: 
Table 1. Node Status

\begin{tabular}{cl}
\hline Classification of Node Status & \multicolumn{1}{c}{ Node Status } \\
\hline Node Status One & Health Status (Susceptible ) \\
Node Status Two & Infected Status (Infected $)$ \\
Node Status Three & Immune Status (Re moved) \\
\hline
\end{tabular}

(2) Propagation model of stochastic immune mechanism

The transformation relations of the three node statuses are presented in Figure 2:

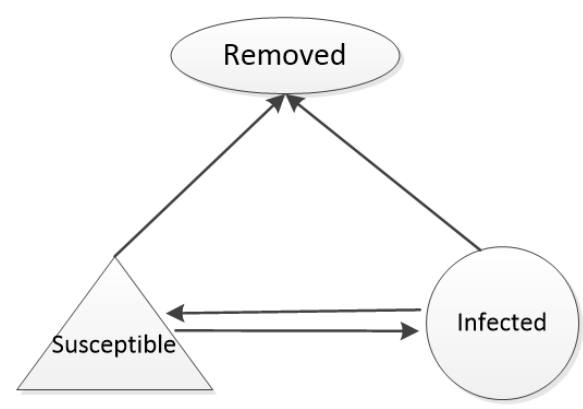

\section{Figure 2. Stochastic Transformation}

If any risk exists in the complicated network could be discovered from the transformation relations of the node statuses, Figure 1 shows the state of risks, that is health status ( Susceptible), namely the risk state equals to 0; infected status (Infected) means security risk existing in the node, namely the risk state is bidder than 0 ; immune status (Removed) means no security risk existing in the environment of node, namely the infection problems of node have been avoided or prevented by relevant immune measures so as to realize the immune status[3]. The model constituted by these three node statuses is named as Propagation Model of Node's Stochastic Immune Mechanism, shortened to as $R-S I R-R$ Model.

$R$-SIR- $R$ Model is established on the basis of stochastic immune mechanism theory and the building mechanism of SIR model. The preconditions of the model establishment are presented as:

(1): at the starting stage of the propagation, every node shares the same propagation probability under the complicated network;

(2): the SIR Model acts as the reference model for the building of new models;

(3): in the condition of immunization, every node shares the same probability of immunizing after the avoidance or prevention of infection;

(4): the node may appear to be immunized, uninfected or healthy after immunizing[4].

Combining the former study achievements with practical situations, the S-SIR-R Model is established as follow:

Given the three statuses of ( Susceptible), (Infected) and (Re moved), namely nodes' three statuses of health, infected and immune, the R-SIR-R Model can be regarded as the Set $M=\{(0,0),(1,0),(0,1),(1,1)\}$, among which the four elements refer to the three statuses respectively[5]. The State Variables of Node $i$ in Time ${ }^{t}$ are signified by the 
Vector Quantity $B_{i}(t)\left(B_{i}(t) \in Q\right)$. While the Vector Quantity $B_{i}(t)$ is $B_{i}(t)=\left(B_{i x}(t), B_{i y}(t)\right)$, which includes $s_{i x}(t)$ and $s_{i y}(t)$, and the $s_{i x}(t)$ represents the infection or uninfected status of the node in this part, while the $s_{i y}(t)$ shows that nodes in this part are immunized, hereafter leads to:

$$
B_{i}(t)=\left\{\begin{array}{llll}
(0,0), & i & t & \text { Susceptible } \\
(1,0), & i & t & \text { Infected } \\
(0,1), & i & t & \text { Removed } \\
(1,1), & & & \text { None }
\end{array}\right.
$$

In the R-SIR-R Model, Time ${ }^{t}$ refers to the related links between adjacency matrix and $C(t)$ in the complex network so as to signify the relationships [6] of nodes in the Time $t$. Among them the Vector Quantity of the $i$ line in the Matrix $A(t)$ represents that the neighbor Set Matrix of this node in the Time $t$ is $N(t)$, namely $N_{i}(t)=\left\{a_{i j}(t) \mid a_{i j}(t) \in C(t), j=1,2 \ldots ., N\right\}$. Among them, when $a_{i j}(t)=1$, at the Time $t$ Node $i$ links with the network risk.

The Transfer Function $f$ of three statuses was defined as: the constitution of transfer rules of three statuses in the $R$-SIR-R Model by two major sections. Firstly, when the Time $t=0$, namely in the initial status condition, every node would be stochastically immunized [7] at the same Probability $y$ in the complex network.

The transformation rules of statuses are presented as follow:

$$
\begin{aligned}
& B_{i x}(0)=0 \\
& B_{i x}(0)=\left\{\begin{array}{l}
1, g>0 \\
o, g \leq 0
\end{array}\right.
\end{aligned}
$$

The $g$ in the formula refers to the judgment function of statuses transformation, which is:

$$
g=\gamma-r
$$

$\gamma$ refers to the random number generated in the range of $(0,1)$, its function lies in the figure comparison with Immunization Rate $y$ to generate the criterion for whether the Node $i$ shall be taken into immune conversion or not.

The function of Formula (1) and Formula (2) lies in the judgment of whether the Node $i$ has been immunized in the state of trouble. In the Formula (2)

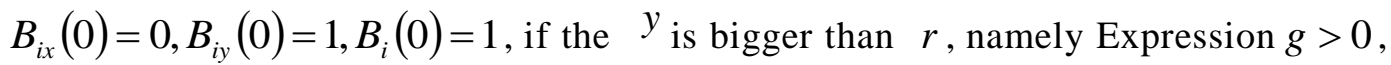
the Node $i$ has been immunized and it is in the status of immunize. However, if $y \leq r$, namely the Expression $g \leq 0$, the Node $i$ is in the condition of pre-immune and it is in the status of health. $B_{i x}(0)=0, B_{i y}(0)=0, B_{i}(0)=(0,0)$

Given the evolutionary stage of the secure network's risk propagation, the thesis further puts forward the upgrade transformation rules to apply to the state of $t>0$. In the evolutionary stage of the security risk propagation of immune mechanism under complex network background, presumptions are proposed as follow:

(1): Node $\delta$ is affecting its neighbors in the Probability $\beta$ at any time period;

(2): Node $\delta$ is recovering in the same probability at any time interval; 
(3): If the infected node would recover at next time period, then at this time period, the node gets immunized [8] in the Probability of $\beta$.

The upgrade transformation rule is presented as follow;

$B_{i s}(t+1)=\left\{\begin{array}{l}\overline{B_{i x}(t)}, g_{x}>0 \wedge B_{i y}(t)=0 \\ B_{i x}(t), g_{x} \leq 0 \wedge B_{i y}(t)=0 \\ 0, B_{i y}(t)=1\end{array}\right.$

$B_{i y}(t+1)=\left\{\begin{array}{l}1, g_{y}>0 \vee B_{i y}(t)=1 \\ 0, g_{y} \leq 0 \wedge B_{i y}(t)=0\end{array}\right.$

If the Node $i$ is in immunize status at Time $t$, there exists $B_{i}(t+1)=(0,1)$, namely the node would stay immunization in later state once it is immunized. While if the Node $i$ is infected in Time $t$, there exists $B_{i}(t+1)=(1,0)$, namely the node is infected to be in the state of infection [9].

$g_{x}, g_{y}$ refers to judgment function of the status transformation, and the computing method is presented as follow:

$$
\begin{aligned}
& g_{x}=\overline{B_{i x}(t)}\left(1-(1-a)^{m_{i}(i)}-\gamma\right)+B_{i x}(t)(\beta-\gamma) \\
& g_{y}=B_{i s}(t) \overline{B_{i x}(t+1)}(\delta-\gamma)
\end{aligned}
$$

The function to signify the number $m_{i}(t)$ of infected nodes neighboring Node $i$ in Time $t$ in the Formula (4) is:

$$
m_{i}(t)=\sum_{j=1}^{N} a_{i j}(t) B_{j x}(t)
$$

Moreover, if $B_{i}(t)=(0,1)$, then $a_{i j}(t)=a_{j i}(t)=0, j=1,2, \ldots . N$

The state of Node $i$ can be judged in the Formula (5), if the node is infected in Time $t$ and will turn to health in next time period, then the node has been immunized and is in immune state now, among them figure $r$ randomly selected in the range of $(0,1)$ is supposed to compare with $\delta^{[10]}$.

If $B_{i}(t)$ equals to $1, B_{i x}(t+1)$ equals to 0 , and $(\delta-r)>0, g_{r}>0$ in the Formula (5), namely the Node $i$ is infected in the time $t$, and it will get immunization and turn to health in the time $t+1$. Beyond that, all other statuses suppose to be $g_{y} \leq 0$, which means Node $i$ is in the pre-immune state.

$B(t)$ refers to the ratio of healthy nodes to the total nodes at the time $t$ in the complex network system, $P(t)$ refers to the ratio of infected nodes to the total nodes at the Time $t$ in the complex network system, $E(t)$ refers to the ratio of immunized nodes to the total nodes at the Time $t$ in the complex network system, the computing methods of the ratio are:

$$
\begin{aligned}
& P(t)=\frac{1}{N} \sum_{i=1}^{N} B_{i s}(t) \\
& E(t)=\frac{1}{N} \sum_{i=1}^{N} B_{i y}(t)
\end{aligned}
$$




$$
B(t)+I(t)+R(t)=1
$$

\subsection{Propagation Model of Acquaintance Immune Mechanism}

(1) Acquaintance immune mechanism

Acquaintance immune mechanism refers to the selection of some specific-proportion nodes stochastically in the complex network system, and then the immunization of one neighboring nodes randomly chosen from the above-mentioned nodes. The introduction of the acquaintance immune mechanism into this model will also lead to the three statuses of node mentioned above, and the transformation relations of this three statuses is presented in Figure 3:

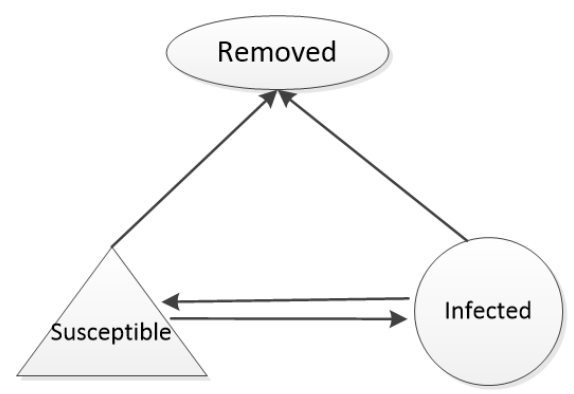

Figure 3. Acquaintance Immune Transformations

(2) Propagation model of acquaintance immune mechanism

The Figure shows that the meaning of the node's status remains the same as in the $R$-SIR- $R$ Model after the introduction of acquaintance immune mechanism. In this designing, the model is defined as propagation model of acquaintance immune mechanism, namely $R-S I R-A$ Model. The $R-S I R-A$ Model is established by the combination of SIR Model mentioned by the previous scholars in biological epidemiology with the propagation model of immune mechanism mentioned above, under the presumptions presented as follow:

(1): The complex network would be immunized when the time equals to 0 , and as the node recover, the node will be immunized at a certain probability based on the SIR Model.

(2): The immunized nodes would not be infected, nor infect the healthy node, therefore, after the node being immunized, its connected edges shall be deleted from the complex network system.

On the basis of cellular automation model building, the $R-S I R-A$ Model is established in accordance with the four model elements therein. The $C, Q$, and $V$ in the model shares the same definitions as in the $R-S I R-A$ Model. Then the Status Transformation Rules $f$ in the $R-S I R-A$ Model will be defined into two aspects as follow:

Firstly, if time equals to 0 , some nodes will be selected from the complex network system in the probability $P$ stochastically, and then one random neighboring nodes chosen from the selected nodes will be immunized.

The Status Transformation Rule $h_{i}$ of the first section is presented as below:

$q_{i}=\left\{\begin{array}{l}1, P-r>0 \\ 0, P-r \leq 0\end{array}\right.$ 
Firstly, the rule of each definition is as follow:

$q_{i}=1$ Means that the node $i$ is chosen by the probability $P$, while $q_{i}=0$ means that the node $i$ is not chosen by probability $P$. And acting as the unknown number stochastically selected in the range of $(0,1), r$ is supposed to compare with the Probability $P$ to assure whether the Node $i$ is chosen or not.

$q_{i}=1$ Means that the Node $i$ is chosen and a neighboring node of Node $i$ will be selected for immunizing treatment. Therefore, the definition of Cellular Field $N$ in the model could be shown as:

Vector Quantity $N_{i}(0)=\left\{a_{i j}(0) \mid a_{i j}(0) \in A(0), j=1,2 \ldots, N\right\}$ refers to the neighboring condition of Node $i$ with other nodes when the time equals to 0 , Set $v_{i}(0)$ refers to the neighboring set of Node $i$ when the time is $0 . s_{i}(0)=\left\{j \mid a_{i j}(0)=1, a_{i j}(0) \in A(0)\right\}$. If $s_{i}(o)=\phi$, the next node of Node $i$ shall be judged to see if it is chosen or not. If $s_{i}(o) \neq \phi$, one element $j$ shall be selected stochastically to be immunized so as to

$q_{i}=0$ Means that the $i$ is not chosen, and the judgment shall be continued to next node.

If $t>0$, there exists following presumptions:

(1): In the process of evolution, infected node is infecting its neighbor nodes in the Probability making the $B_{j r}(0)=(0,1) . a$ at any tome period;

(2): Infected nodes are recovering and getting immunized in the Probability $\beta$ at any time period.

$R-S I R-A$ Model shares the same status transformation with $R-S I R-R$ Model.

$B(t)$ Refers to the ratio of healthy nodes to the total nodes at the Time $t$ in the complex network system, $P(t)$ refers to the ratio of infected nodes to the total nodes at the Time $t$ in the complex network system, and $E(t)$ refers to the ratio of immunized nodes to the total nodes at the Time $t$ in the complex network system. $R-S I R-A$ Model shares the same results of Formula (7),(8) and (9) with $R-S I R-R$ Model, which is:

$$
\begin{aligned}
& P(t)=\frac{1}{N} \sum_{i=1}^{N} B_{i s}(t) \\
& E(t)=\frac{1}{N} \sum_{i=1}^{N} B_{i y}(t) \\
& B(t)+I(t)+R(t)=1
\end{aligned}
$$




\section{Evolutionary Analysis of Security Risk Propagation under Complex Network Background}

(1) Evolutionary Analysis of Security Risk Propagation

This section emphases on the security risk propagation analysis of the model introduced immune mechanism. Figure 4 and Figure 5 describe the security-risk propagating trend and affecting scale of the $R-S I R-R$ Model and $R-S I R-A$ Model in the networks of $N C, E R, W S$ and $B A$ respectively, and the parameter relations are as follow:

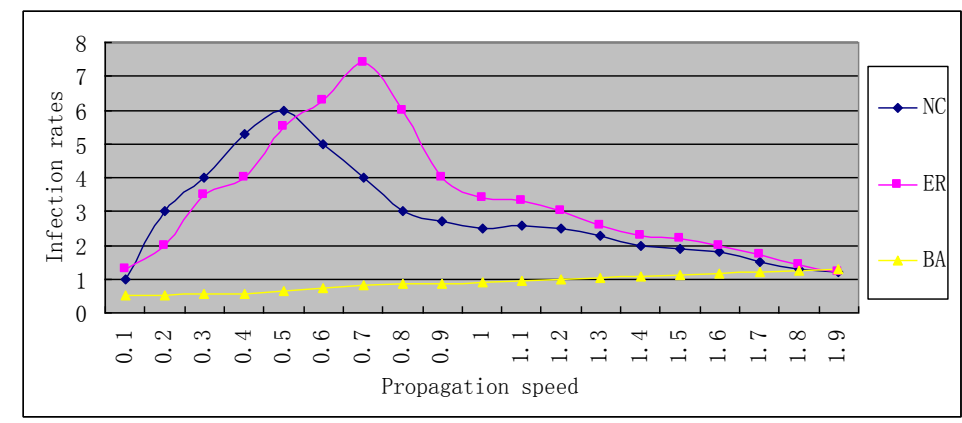

Figure 4. The Propagating Trend and Affecting Scale of R-SIR-R Model in the NC Network, ER Network, WS Network and BA Network

Figure 5.The Propagating Trend and Affecting Scale of R-SIR-A Model

Given consideration to the immune mechanism, the security risk propagating trend of $R-S I R-R$ Model and $R-S I R-A$ Model presented by Figure 3 and Figure 4 shows that the risk dies out in a slow speed in the $N C$ Network, which shows that the immune mechanism could control the influence of $N C$ Network's security risk in a very small scale.

The principle is that the immune mechanism simulates the $N C$ network into a community network in the model, whose structure is featured by internal links counting a major part while external links a small part of the network. Therefore, the difficulty of security risk propagation contributes to the easy-control of the security risk. However, it is difficult to eliminate the security risk as soon as possible due to the common existence and consistent propagation of the security risks in every community.

In other three network topological structures of $B A, E R 、 W S$, the speed of risk elimination is $B A<E R<W S$, namely an increasing order in accordance with network topological structures of $B A 、 E R 、 W S$. This situation mainly due to that the higher the heterogeneity degree distributed in the complex network degree is, the higher the possibility of immunization realized by the immune mechanism to higher-degree nodes will be. However, as for the extremely high-degree node, which would be easily infected by neighbor nodes and infect its neighbor nodes, will significantly influence the 
propagation of security risk.

This thesis will continue to analyses the influence of three different community network topologies on the security risk propagation. Figure 6 describes the propagating trend and affecting scale of security risk of $R-S I R-R$ Model in different community network topologies. Among which, the parameters are presented as follow:

$$
(\alpha=0.3 ; \beta=0.8 ; \gamma=0.01 ; \delta=0.1 ; K(0)=2 / N) \text {. }
$$

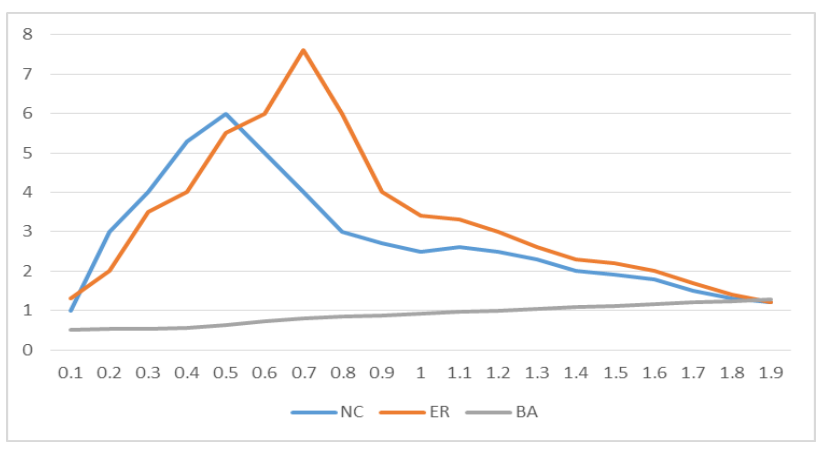

\section{Figure 6. The Propagating Trend and Affecting Scale of R-SIR-R Model in Different Community Networks}

Figure 6 shows that the propagation speed of security risk of $R-S I R-R$ Model is $N C>E R>B A$, which leads to the conclusion that the lower the connectivity probability of inner community and inter community in the network topology is, the higher the propagation speed of security risk will be. All the infection rates of three networks are approach to 0 finally, which means that the stochastic immune mechanism of $R-S I R-R$ Model resulted in a sound control over the security risk propagation.

(2) The die-out analysis of security risk propagation in initial stage under the complex network background

The thesis analyses the die-out situation in the initial stage of the security risk propagation models of stochastic immune mechanism and acquaintance immune mechanism, as well as the relationships between the die-out rate and propagating rate at the initial stage of security risk propagation. The Figure 7 and Figure 8 show the relation curves between the die-out rate and Propagating Rate $\lambda$ at the initial stage of security risk propagation under the $R-S I R-R$ Model and $R-S I R-A$ Model respectively. Among which, the parameters of Figure 7 is $(y=0.01 ; \delta=0.1 ; K(0)=2 / N)$; , and the parameters of Figure 8 is $(p=0.01 ; \delta=0.1 ; K(0)=4 / N)$ 


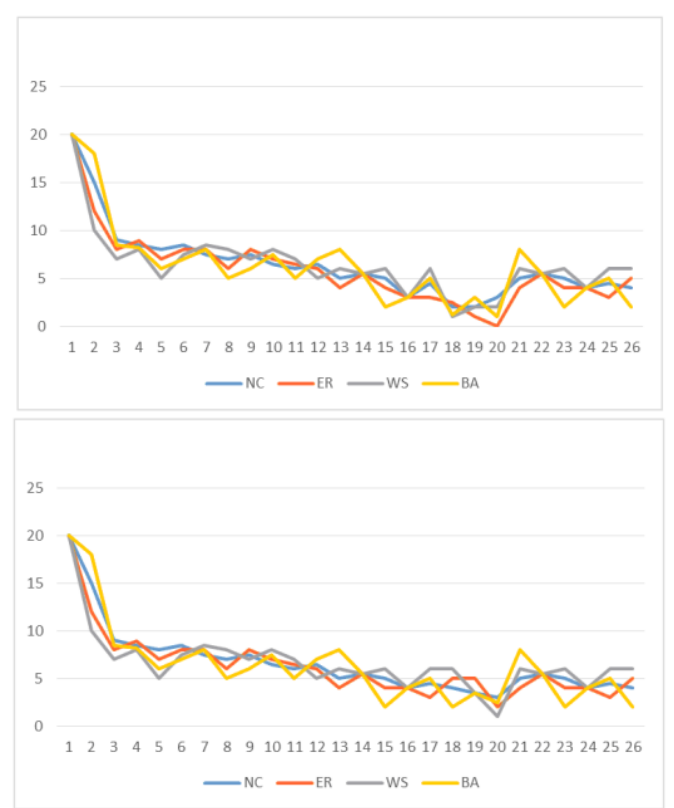

Figure 7. Relation Curves Between the Die-out Rate and Propagating Rate at the Initial Stage of Propagation under R-SIR-R Model

Figure 8. Relation Curves Between the Die-out Rate and Propagating Rate at the Initial Stage of Propagation under R-SIR-A Model

The Figure 7 and Figure 8 present the die-out condition of the security risk at the initial stage of propagation and also lead to the conclusion that the die-out rate is inversely proportional to the propagating rate.

As for the die-out situation of security risk at the initial stage of propagation, the thesis reaches to the conclusion that the smaller the Probability $a$ is, the bigger the Probability $\beta$ will be , namely the bigger the die-out rate will be. Due to the Propagating Rate $\lambda=\alpha / \beta$, it could be concluded that with the increase of propagating rate, the die-out rate of security risk at the initial stage of propagation will decrease. By means of 
increasing the rate $P(0)$, which refers to the rate of infected nodes to all the nodes, in different Time $t$ in the complex network system, Figure 7 and Figure 8 could also concluded that the $P(0)$ in different time periods is inversely proportional to the die-out rate.

\section{Conclusion}

The thesis probes into the safe propagation of complex network after the adoption of immune mechanism. On the basis of introducing propagation models of stochastic immune mechanism and acquaintance immune mechanism, the thesis analyses the security risk propagation and evolution of models under various kinds of network topological structures by means of simulation, and the thesis establishes models directly in accordance with the rates of infected nodes and healthy nodes to the total nodes respectively at different time periods in the complex network system. The establishment of immune mechanism model leads to the conclusion that the $P(0)$ is inversely proportional to the die-out rate. Therefore, the immune mechanism would contribute to the reduction of complex network risk issues and the decrease of the security risk rate in network propagation.

\section{References}

[1] Cao Q., Lai H. and Ge W. Q., "Research and Development of Mobile Termination for the Steel Quality Evaluation System Based on Android[C]", //Applied Mechanics and Materials, vol. 513, (2014), pp. 960-964.

[2] Gammer N., Cherrett T. and Gutteridge C., "Disseminating real-time bus arrival information via QRcode tagged bus stops: a case study of user take-up and reaction in Southampton", UK[J], Journal of Transport Geography, vol. 34, (2014), pp. 254-261.

[3] Hildyard K. L. and Wolfe D. A., "Child neglect: developmental issues and outcomes [J]", Child Abuse Neglect, vol. 26, no. 6-7, (2002), pp. 679-695.

[4] Julie A. S., Amy M. and S. Slep, "Risk factors for child neglect", Aggression and Violent Behavior, vol. 6, (2001), pp. 231-254.

[5] J. Kim, M. S. Lee, A. Yun, "CRT-based Fully Homomorphic Encryption over the Integers[J]", IACR Cryptology Print Archive, vol. 57, (2013).

[6] E. C. Iliff, "Computerized medical diagnostic and treatment advice system including network access: U.S. Patent 9", 005, 119[P], (2015) April 14.

[7] Marwick C., "Domestic violence recognized as world problem", JAMA, vol. 279, no. 19, (1998), p. 1510.

[8] Mell P. and Grance T., "The NIST Definition of Cloud Computing(draft) [M]", NIST Special Publication, (2011).

[9] Raju R, Amudhavel J. and Kannan N., "Interpretation and evaluation of various hybrid energy aware technologies in cloud computing environment-A detailed survey[C]",//Green Computing Communication and Electrical Engineering (ICGCCEE), 2014 International Conference on, IEEE, (2014), pp. 1-3.

[10] Schumacher J. A., Smith Slep A. M. and Heymanl R. E., "Risk factors for child neglect[J]", Aggression and Violent Behavior, vol. 6, (2001), pp. 231 -254.

[11] Straus M. A., "Identification of child maltreatment with the Parent-Child Conflict Tactics Scales: development and psychometric data for a national sample of America parents", Child Abuse \& neglect, vol. 22, (1998), pp. 249-270.

[12] Tanner K. and Tumey D., "What do we know about child neglect? A critical review of the literature and its application to social work practice", Child and Family Social Work, vol. 8, no. 1, (2003), pp. 25-34. 


\section{Author}

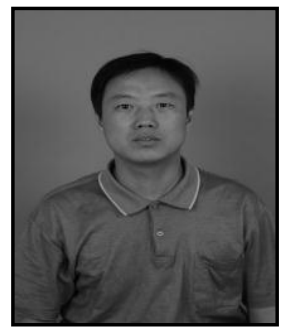

Lu Cailin. He is an associate professor and master of the School of Information Technology and Engineering in Jinzhong University. He teaching and researching computer network and computer simulation. The address of his working place is 199 Wenhua Street, Yuci District, and Jinzhong City, Shanxi Province. 